Down Syndrome Research and Practice Vol 5, No.1, pp 16 - 25

(C) The Down Syndrome Educational Trust

Printed in Great Britain. All rights reserved.

ISSN: 0968-7912 (1998) 5:1

\title{
USING AUGMENTATIVE COMMUNICATION WITH INFANTS AND YOUNG CHILDREN WITH DOWN SYNDROME
}

\author{
Phil Foreman \\ Special Education Centre \\ Geoff Crews \\ Department of Psychology \\ University of Newcastle, NSW, Australia
}

\begin{abstract}
This paper reports the use of two forms of augmentative and alternative communication (AAC) with young children with Down syndrome: a program using signing (Makaton), and the COMPIC system of computerised pictographs. Children with Down syndrome are frequently reported to have difficulties in the area of language and communication, with relative strengths in visual and perceptual areas. This suggests possible benefits from the use of AAC systems to enhance language development. The paper discusses the use of AAC systems to assist young children with Down syndrome, and reports an experimental study of the use of such systems with an object naming task.
\end{abstract}

Keywords: Down syndrome, infants, children, augmentative communication, sign, symbols

The term augmentative and alternative communication (AAC) is used most frequently to refer to mechanical and electronic systems that give communicative access to people with cerebral palsy and other physical disabilities (Smithers \& Puffett, 1996). The other common AAC system has been the use of sign language within the deaf community. However, during the past 20 years, both literature and practice suggest that sign language has changed from being used solely as an alternative communication for the deaf community, to one which can be used to augment communication for persons who have some other impairment to standard communication (Bricker, 1972; Creedon, 1976; Hobson \& Duncan, 1979; Konstantareas, 1985; Iacono \& Parsons, 1987; Weisel, Dromi, \& Dor, 1990).

Following the early and widespread use of language systems such as Margaret Walker's 'Makaton Vocabulary' (Walker, 1978), which was developed as the basis of a signing program for adults and older children with developmental disabilities, it became evident that there may be value in using signing with young children who are known from birth to be at risk of being developmentally delayed, such as children with Down syndrome (Walker, 1978; Prevost, 1983). This group of children was originally targeted because studies of children with Down syndrome of school age have suggested that the most common problems lay mainly in aspects of speech and language (Carr, 1975). On the other hand, motor and visual perceptual skills are an area of relative strength for young children with Down Syndrome (Kumin, 1994; Wang \& Bellugi, 1994). Thus, early attempts to introduce inter-related speech and sign, before speech had even developed, were expected to alleviate some of the problems encountered, and influence the pattern of speech and language development. A natural progression from the use of Makaton signing with babies and toddlers with Down Syndrome has been its extension to use with other young children with developmental 
disabilities and for whom it is suspected that verbal language will be delayed.

Parallel to the introduction and use of signed visual systems to augment communication has been the development of pictorial representation systems. These have included Bliss and Rebus and, more recently, COMPIC and Picture Communication Symbols (PCS). COMPIC is a communication resource that does not rely on reading the written word, hearing a word, or speaking a word. Rather, it comprises some 1700 clear and easily understood drawings, or pictographs, which convey information.

Not surprisingly, although research has reported positive outcomes from the use of augmentative systems such as Makaton and COMPIC, several researchers have highlighted the reluctance of some parents to approve or use signing with their children (Berry, 1987; Iacono \& Parsons, 1987; Parsons \& Wills, 1992). These studies suggest that parents have some of the following concerns:

(1) fear that using an augmentative system will hinder and/or prevent the development of verbal language;

(2) introduction of augmentative communication is an indication of "giving up" on verbal expression;

(3) the stigma of using augmentative communication in a speaking world; and

(4) the perceived difficulty for the child and family to "learn" the augmentative system, particularly signs.

However, research studies, together with feedback from parents whose children have successfully used augmentative systems, suggest that the opposite is actually true for all of the above concerns (see Parsons \& Wills, 1992, for a review). They further suggest that, without augmentative communication, children with speech/language delays, who can often understand much more than they can verbally express, sometimes become very frustrated and resort to unacceptable means of communication. These might include tantrums and screaming, or giving up the effort to be understood. Kumin (1994) suggests that by enabling a child to communicate, augmentative communication also reinforces basic language concepts, while empowering the child to influence his world, until he is able to do so verbally.

\section{Augmentative communication within the Early Education Program at the University of Newcastle, Special Education Centre}

The Early Education Program at the University of Newcastle Special Education Centre began in 1978 and has been using Makaton signing since 1983. The use and outcomes of Makaton within the program have fairly closely mirrored the findings of research. That is, as acceptance of augmentative communication with infants, toddlers and young children has increased over time, so too the use of Makaton and more recently COMPIC, has grown within the program. Positive outcomes to date have generally been determined via parent feedback, feedback from relevant others, as well as observation and evaluation of the child's individualised program.

The Makaton Vocabulary relies heavily on natural gestures, body language and facial expression to portray the messages. This appears to enable children and adults to master the signs quickly. COMPIC is used via visual timetables and/or communication cards or boards. The selection and maintenance of a system for a child is suggested after observing the child's learning preferences and abilities, as well as through discussion with the family regarding their preferences. Generally it is found that most of the young children with Down syndrome cope well with a combination of Makaton signing and COMPIC.

Previously only used by a small number of children, and even then only after two to three years of age, these systems are now used for all the children enrolled in the Early Intervention Program at the Special Education Centre. Makaton is introduced to children as young as 7-8 months, with the expectation that peers will gradually be able to interact and communicate with each other, as well as with the teachers in the program. Signs are practised at every opportunity and taught in both a formal and an informal manner within the groups. Many of the signs are learned through fingerplays and songs.

The selection of the vocabulary taught to the children is user-friendly and functional. The first signs introduced are more, finished, help and no. The naturally occurring gestures such as hello, good-bye, go and stop are usually already in place. These initial signs (more, finished, help and no) are powerful signs for the children to use. Through these signs, they can request assistance, continue an activity, or terminate an event. The aim is for children to be empowered by a communication system which can assist them throughout their day. Other words chosen need to be functional and easy to form. Words which are not found in Makaton have been taken from the Auslan (Australian Sign Language) dictionary. Only key words are signed to the children, and normal speech is always used concurrently. There is a list of approximately 160 words which is given to families as the most commonly-used words spoken in the Centre. The 
list is negotiated and contains words which are specific to the target child.

COMPIC is introduced when the child shows some ability to respond to more abstract stimuli, usually at two years plus. It is used throughout the program in many ways. Nouns, verbs and adjectives on communication boards, lotto games, stories and visual timetables are the main ways in which COMPIC is used. The progression is usually: object ${ }^{`}$ picture ${ }^{\sim}$ COMPIC.

\section{Children with significant difficulties with} transitioning, attention to task, or with choosing an activity, appear to have made gains when using a visual scheduling system which incorporates COMPIC. The COMPIC program usually begin with choice boards and objects or photos, and then progresses from the schedule boxes to schedule sheets.

The evaluation of the effect of different teaching programs on the way young children with disabilities acquire language is not easy, due to the number and interrelationship of variables involved. The present study was an attempt to control as many of these variables as possible through an experimental study of the way in which young children with Down syndrome learn the names of objects with the assistance of augmentative communication techniques.

\section{Method}

The study utilised a simple repeated measures technique. All children who participated were encouraged to learn to communicate 12 single words: three via verbal instruction alone, three via the symbol (COMPIC) method, three via the sign (Makaton) method, and three via the multimodal method (verbal + sign + symbol). The four treatments (verbal, symbol, sign and multimodal) were administered successively over four days, with interaction and sequence effects being controlled for by a Latin square design. As all participants learnt the same words (via different methods), and participated in all treatments, each participant was his or her own control (Benjamin, 1965).

Learning in this study was assessed according to the relearning (or savings) strategy. The relearning measure of retention requires a participant to memorise information a second time to determine how much time or effort is saved by having learned it before. In contrast to a pass/fail criterion, the relearning technique is sensitive in detecting small amounts of information in memory. Savings scores can vary to take into account relearning efficiency, whereas pass/fail tests are usually of an all-or-none character (Bahrick, 1967; Nelson, 1978).
Apart from allowing participants' performances to be scored, the relearning strategy also allowed participants' scores to be plotted over time. Participants participated in the learning tasks in this study on three separate occasions: (1) initial teaching phase, (2) 15 minutes later, and (3) 24 hours later. As participants' scores would be expected to increase with repeated teaching sessions, their learning patterns over time enabled a check of whether genuine learning had occurred.

\section{Participants}

Participants were 19 children (8 girls) with Down syndrome. All children were aged between 2 and 4 years and had been involved in an early intervention/special needs program for at least six months. Inclusion of children in the study was dependent upon parents' informed consent.

\section{Procedure}

All participants were shown the same 12 objects. However, if a child was able to communicate the name of an item prior to instruction (during the pretest), the object was replaced with one of four spare items. Altogether, 16 stimuli were used in the experiment. The 16 symbols for the experiment were taken from the COMPIC (1994) communication resource. All symbols were laminated black-line drawings on a white paper background measuring $15.5 \mathrm{~cm} \times 11.5 \mathrm{~cm}$. The signs were from two sources: the Dictionary of Australasian Signs (Jeanes, Reynolds, \& Coleman, 1993) and the Revised Makaton Vocabulary (Walker, Johnston, \& Cornforth, 1984).

Each of the treatments consisted of three teaching sessions: the initial teaching session; a postteaching session 15 minutes later; and a follow-up teaching session 24 hours later. Each session comprised a maximum of four practice trials. Employing the relearning strategy, the number of timed practice trials (of approximately 40 seconds) the child needed to communicate each word appropriately was recorded. As each participant was taught each word on three occasions (over the three sessions), how much more quickly the child communicated the word in the second and third sessions constituted his or her savings scores. These scores were the dependent variable of this study.

Each day, the child was taught three words via one of the four communication methods. Prior to teaching, the tester (GC) checked that the child did not already know how to communicate the new words for that day. This was done via a pretest, and confirmed by the parent.

A child was said to have learnt a word when 
he/she could communicate the word, without help, after being prompted. Deciding whether a participant had successfully communicated a word was decided by a previously determined classification system, which is detailed under data analysis.

Participants were instructed/tested in their own homes, usually in the presence of a parent. All instructions and procedures were administered to each child by the second author.

\section{Data collection, scoring, and analysis}

Video recordings of sessions were taken for later scoring and reliability rating. On completion of data collection, the videos were analysed and all participants were scored on their ability to communicate the words they were taught. Three trained early education teachers viewed random samples of these videos and also scored the participants' ability to communicate. A check for inter-rater reliability was performed using Kendall's coefficient of concordance (Ferguson, 1959).

A child received a score of 4 if, after being shown an object and asked 'what's this', he/she could communicate the word appropriately within the 40 second trial. A score of 3 would be awarded if the child needed to be shown how to communicate the word before he/she could communicate it on request. Similarly, 2 points would be awarded if the child needed to be shown twice, and 1 point if the child needed to be shown how to communicate the word three times (three 40 second trials) before being able to do so on his/her own, upon request. If the child did not learn by this third prompting, then he/she was taught the next word for that session and was awarded no points for the word on that occasion.

As noted, each participant learnt three words via each of the communication methods. This procedure was utilised in an effort to minimise the possibility of a participant fortuitously "communicating" and thereby contributing an artificial learning effect. For the purposes of data analysis, participants' scores for these three words were averaged to give each participant one score per method, per session. As each child participated in four communication methods across three sessions, they each contributed 12 scores (each out of 4) to the data analysis.

\section{Results}

Inter-rater reliability was investigated using Kendall's coefficient of concordance. Employing an alpha level of .05 , a chi-square analysis indicated a significantly high level of concordance between the three special education teachers and
Figure 1. Subjects' mean scores for the four methods across three learing sessions.

the tester.

Table 1 shows that the Verbal method of instruction produced no successful trials. However, mean scores increased across the Symbol, Sign and Multimodal methods respectively, and across the three sessions. The mean scores for each method were then plotted across the three sessions, as illustrated in Figure 1. The figure shows the four methods of instruction to have a relatively consistent increasing effect upon subject scores
Table 1. Descriptive statistics of scores for four methods at three sessions.

\begin{tabular}{lcccccc} 
Variable & $N$ & Mean & Median & StDev & Min & Max \\
Session1 & \multicolumn{7}{l}{} & & & & & \\
Verbal & 19 & 0.000 & 0.000 & 0.000 & 0.000 & 0.00 \\
Symbol & 19 & 1.368 & 1.333 & 0.962 & 0.000 & 3.00 \\
Sign & 19 & 1.632 & 1.333 & 1.133 & 0.000 & 3.00 \\
Multimodal & 19 & 1.702 & 1.667 & 1.122 & 0.000 & 3.00 \\
Session 2 & (15 & minutes after Session 1$)$ & & & \\
Verbal & 19 & 0.000 & 0.000 & 0.000 & 0.000 & 0.00 \\
Symbol & 19 & 1.807 & 2.000 & 1.484 & 0.000 & 4.00 \\
Sign & 19 & 2.175 & 2.333 & 1.450 & 0.000 & 4.00 \\
Multimodal & 19 & 2.404 & 2667 & 1.408 & 0.000 & 4.00 \\
Session 3 (24 hours after & Session 1$)$ & & & \\
Verbal & 19 & 0.000 & 0.000 & 0.000 & 0.000 & 0.000 \\
Symbol & 19 & 1.930 & 1.667 & 1.289 & 0.000 & 4.00 \\
Sign & 19 & 2.491 & 2.667 & 1.316 & 0.000 & 4.00 \\
Multimodal & 19 & 2.491 & 2.333 & 1.316 & 0.000 & 4.00
\end{tabular}

across the three sessions. 
Table 1 indicates that the Verbal method of instruction, resulting in consistent zero scores, contained no variability. Because of this, and the lack of need to statistically investigate this finding, scores for the Verbal method were eliminated from further analyses conducted on the data.

\section{Results of the $3 \times 3$ ANCOVA}

Statistical analysis therefore involved a $3 \times 3$ ANCOVA (three Session levels: initially, 15 minutes later, and 24 hours later; by three Method levels: Symbol, Sign and Multimodal). Subject variables were entered into a mixed design, with age and gender treated as covariates, and Method and Session treated as within subject factors. Children's scores were the dependent variable for the study.

The mixed design analysis found a significant main effect of method

$[\mathrm{F}(2,19)=3.53, \mathrm{p}=.04]$ and session

$[\mathrm{F}(2,19)=14.44, \mathrm{p}=.0001]$, and

a non-significant interaction between the two $[\mathrm{F}(4$, 19) $=.43, \mathrm{p}=.79]$.

The main effect of method was further investigated using a univariate F-test. This found the Sign method of instruction to invoke significantly higher scores than Symbol instruction $[\mathrm{F}(1,18)=8.362, \mathrm{p}=.01]$. Scores obtained for the Multimodal method of instruction, although obviously significantly higher than Symbol instruction, were not shown to be significantly higher than those scores for Sign instruction $[F(1$, 18) $=.607, p=.446]$.

In agreement with the above F-test, three onesample t-tests found scores obtained for the Multimodal method of instruction to be nonsignificantly different from scores obtained for Sign instruction. This was the case across all sessions: Session $1[\mathrm{t}(19)=0.27, \mathrm{p}=.79]$, Session $2[\mathrm{t}(19)=0.71, \mathrm{p}=.48]$, and Session $3[t(19)=0.00, p=1.0]$.

Because of this non-significant difference between the scores for the Multimodal and Sign methods, scores for these two methods were averaged. Thus, each child was then assigned a score for the Symbol method and a score for the Sign and Multimodal method combined. A further $3 \mathrm{t}$-tests were performed on the difference between these two scores.

The superiority of both Sign and Multimodal methods over the Symbol method, found in the F test, was also largely supported by three t-tests. Without adjusting for family-wise error, the differences between the averaged Sign-Multimodal method scores and Symbol scores were uniformly significant across the three sessions. However, adjusting for this error increased the required level of significance from .05 to .017 . This resulted in the averaged sign-multimodal method facilitating significantly greater performance than the Symbol method on

Session 3 only:

Session 1, t $(19)=1.98, \mathrm{p}=.05$;

Session 2, $\mathrm{t}(19)=2.13, \mathrm{p}=.037$; and

Session 3, $\mathrm{t}(19)=2.53, \mathrm{p}=.014$.

For multiple t-tests, the family-wise error adjustment was employed only when a group of $t$ tests, pertaining to a single hypothesis, were found to be significant.

Scores for the Multimodal method alone were then analysed. As the Multimodal method gave children the opportunity to respond via one of three methods of communication (verbal, symbol or sign), a chi-square test was conducted to see whether children favoured any one method. As with the other simultaneous methods of communication, verbal language was not used at all. Symbol and Sign communication methods, however, were favoured equally. Of the 124 trials in which children responded to the Multimodal method, 66 responses were via Symbol, and 58 via Sign. This difference was not significant: $\boldsymbol{X}^{2}(1, \mathrm{~N}$ $=124)=0.516, \mathrm{p}>.25$.

Although no mode of communication seemed to be favoured, a two-sample t-test was conducted to investigate whether one of the modes was attained faster ie. received higher scores. This was not found $t(122)=0.18, p=.86$.

Similarly, no significant gender differences were found in children's preferences for a mode of communication $\mathrm{t}(122)=0.76, \mathrm{p}=.45$. Age was also a non-significant predictor of which mode children would choose to respond to when participating in the Multimodal method $\mathrm{t}(122)=0.51, \mathrm{p}=.61$.

A significant main effect of time (session) was found. A further univariate F-test suggested that this difference lay between scores (for all methods) in session 1 and session $2[\mathrm{~F}(1,18)=$ 30.06, $\mathrm{p}<.001]$. Scores obtained in sessions 2 and 3 were not significantly different $[F(1,18)=$ $2.16, p=.159]$. Further t-tests were not conducted on this finding as this research was not concerned with the effect of time, but with the effect of different methods, on learning.

Furthermore, as the interaction between session and method was not significant, simple effects and linear contrasts were not performed. This nonsignificant interaction enabled an unambiguous interpretation of the main effects. 
The covariates of age and gender were not found to be significant predictors of scores, yielding $\mathrm{t}$ scores of $\mathrm{t}(19)=1.680, \mathrm{p}=.112$ and $\mathrm{t}(19)=$ $1.449, \mathrm{p}=1.67$ respectively. However, the direction of these relationships, as indicated by the $B$ values, showed that females tended to score

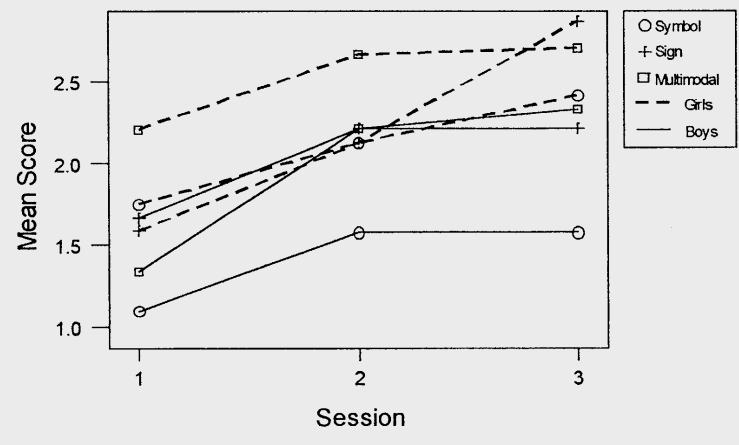

Figure 2. Male and female mean scores for the three methods

As the above t-tests and plots for gender were collapsed across age, a second two-sample t-test was performed on age and gender to investigate the possible confounding effect of age upon these statistics. This t-test found the males to have a significantly higher average age than the females $t(146)=3.71, p=.0003$. Thus the effects of gender may actually be stronger than is evidenced by the above t-tests and plots. However, according to the ANCOVA, the effect of gender (like the effect of age) was not significant.

Since the covariates of age and gender were found to have a non-significant impact upon the scores of children, a repeated measures ANOVA was reperformed on the data (without entering these variables). Again, the main effects of method and session were found to be significant, with a non-significant interaction between them. The statistical values for this analysis were identical to the analysis which included the covariates of age and gender. The conclusions that

higher than males, and older children to score higher than younger children.

A simple effects analysis was then conducted for males and females, using two-way repeated measures ANOVAs. The MSerror terms used in these analyses were taken from the overall analysis conducted on all 19 children. This provided a better estimate of error variance, being based on a larger sample. Although they did not alter the significance of the F scores, the resulting $\mathrm{F}$ scores and $\mathrm{p}$ values, using these MSerror terms, are incorporated into the ANOVA tables.

The ANOVA for females found a significant effect of session

$[\mathrm{F}(2,36)=9.40, \mathrm{p}=.003]$ and the method*subject interaction $[\mathrm{F}(14,72)=3.48, \mathrm{p}=$ .002]. The ANOVA for males found a significant effect of method $[\mathrm{F}(2,36)=4.20, \mathrm{p}=.03]$, session $[\mathrm{F}(2,36)=6.64, \mathrm{p}=.006]$ and the method*subject interaction $[\mathrm{F}(20,72)=2 \cdot 10, \mathrm{p}=$ .022]. These effects are illustrated in the plots for male and female mean scores (see Figure 2).

Although gender was largely found to have a nonsignificant effect on subject performance for each of the methods of instruction, a two-sample t-test revealed that there was a significant difference between male scores and female scores across sessions for all methods combined. That is, females were found to score significantly higher than males overall: $\mathrm{t}(161)=-2.39, \mathrm{p}=.018$. could be drawn from the data were therefore unchanged.

\section{Discussion}

The ability to name objects is of great use to young children with a limited capacity to communicate (Grove \& Walker, 1990). Much of the frustration experienced by young children with communication difficulties is eliminated by the ability to name a desired toy, food, or activity. The present study has taught children naming skills through four methods of communication. The results have suggested a hierarchy of efficiency of these methods in their ability to facilitate such skills for the children involved.

The findings of this study provide support for the practice of using a multimodal augmentative method to enhance communication on a naming task with young children with Down syndrome. As the results have shown, both the simultaneous instruction of verbal and sign language (the sign method), and the simultaneous instruction of verbal, symbolic and sign language (the multimodal method) resulted, on average, in significantly higher scores for all children. This finding is similar to that of Iacono, Mirenda, and Beukelman (1993) and Iacono and Duncum (1995) who found the combined use of unaided and aided augmentative systems to facilitate early communication in children with intellectual disabilities.

The simultaneous instruction of verbal and 
symbolic communication (the symbol method) did not result in scores as high as those produced by the sign and multimodal methods. However, the symbol system did result in significantly higher scores than the verbal-alone method of instruction. None of the children were able to communicate the names of the stimulus words when verbal tutoring was the only method of instruction. This result is consistent with past findings, such as those of Brady and Smouse (1978); Sisson and Barrett (1984); and Greenwald and Leonard (1979). Bonvillian and Folven (1993) suggest that this may be due to the iconic components of both sign and symbol systems, in contrast to verbal language. Other possible explanations are that the visual and motor systems involved in sign and symbol methods mature more rapidly than the corresponding systems in speech (see Bonvillian \& Folven, 1993, for a review).

Although it can be concluded that the multimodal method of instruction is an effective way of encouraging children with Down syndrome (between the ages of two and four) to communicate the names of objects, the results of the present study give no indication of a preferred method that all children will utilise in offering that communication. This contrasts with the conclusions of Iacono and Duncum (1995), whose single subject demonstrated an obvious preference for the aided system (an electronic communication device). Consequently, Iacono and Duncum concluded that, as graphic symbols make less of a demand on working memory (Iacono et al., 1993), they provide greater support for the emergence of communication than does sign.

However, the present study suggests that it is signing that may provide the weight of influence in facilitating communication for these young children. As there was no significant difference between performances for the sign and multimodal systems, it could be concluded that the inclusion of an aided symbolic system into a child's initial communication lexicon will not alter the child's communication performance. However, the fact that approximately half of the responses made for the multimodal system were through symbolic communication suggests that some children prefer to use symbols over signing when presented with both.

These results suggest that there is nothing lost in allowing a child to make his or her own choice concerning a method of augmentative communication. Indeed, this study has found that teaching a child several methods simultaneously results in that child communicating more. As has been previously mentioned, placement of children with speech impairments into augmentative communication systems has recently been an area of considerable interest for researchers in this field. Arthur (1989), for example, has highlighted several flaws in the practices at that time. Since then, a number of decision matrices have been created to encourage careful examination of student characteristics (such as cognitive, motor, intelligibility, imitative and environmental factors) that may contribute to that student's success or failure in using a particular system. Examples include those proposed by Hamre-Nietupski, Nietupski, and Rathe (1986); Kiernan and Reid (1987); Owens and House (1984); and Shane and Bashir (1980). Although the importance of these student characteristics is unquestionable, the present findings suggest that many of the preelection considerations may be put to rest by the students themselves.

As would be expected in experiments involving learning over time, subject performance increased initially, then reached a plateau with increased trials. This finding supports the view that the children in the present study exhibited a genuine learning effect analogous to that of children without Down syndrome. This supports the contention of many researchers that children with Down syndrome learn in similar ways to most other children, the main difference being the rate at which learning takes place (Henderson, 1985; Lenneberg, 1967). Indeed, a number of researchers have found youngsters with Down syndrome to have many communicative functions comparable to the age-matched population of typically-achieving children (eg., Coggins, Carpenter, \& Owings, 1983; Johnston \& Stansfield, 1997; Wetherby, Yonclas \& Bryan, 1989).

Although gender did not have a significant effect on subjects' performances in each of the methods of communication, the data again reflected that females tended to score higher than males.

Additionally, a significant overall gender effect was found. Collapsing across age, for all methods and all sessions, females, on average were found to score significantly higher than males. This supports a number of past studies which have found females with Down syndrome to be superior on general tests of intelligence (Carr, 1988; Sloper, Cunningham, Turner, \& Knussen, 1990).

It is recognised that the nature of the present study limits generalisations about the effectiveness of the methods employed. What this study does provide, however, is a realistic evaluation of a specific communication system for the specific population for whom it was intended. Some young children with Down syndrome may never be able to maintain a 'normal social discourse', particularly at two years of age (Kumin, 1994). Individuals 
with an intellectual disability are likely to experience problems with processing, retention, and recall of information, which limit the amount of language learned, the rate of its acquisition, and the extent to which language is generalised outside formal teaching situations (see Grove \& Walker, 1990, for a review). These factors suggest that, initially, such individuals may benefit from a structured approach which limits the size of vocabulary to be learned and the multitude of applications that it may have (Grove \& Walker, 1990). The initial goal of language acquisition programs, like that of the present study, is to establish basic communication. Once this has been achieved, the student may progress toward comprehensive language use, in whatever mode, or combination of modes, is appropriate. However, even if the child is unable to develop comprehensive language, the ability to communicate basic needs will still contribute to that individual's quality of life (Grove \& Walker, 1990).

There has always been considerable disagreement about the role that imitation plays in the acquisition of language (Taplin \& Lee, 1982). Whitehurst and Vasta's (1975) CIP hypothesis states that it is only when comprehension (C) has been established should selective imitation (I), and thence, spontaneous production (P) take place. As Taplin and Lee (1982) found, however, while it would appear that imitative acquisition is relatively dependent upon comprehension, such comprehension is not absolutely essential to imitation, even of structured sentences.

Consequently, it is possible that some children in this study may have purely imitated the actions they were shown, rather than meaningfully linked those actions to their reward - the named object.

\section{Conclusions}

Numerous studies have emphasised the importance of cognitive, motor, and social prerequisites to communication and language training (Arthur, 1989; Hamre-Nietupski et al., 1986; Kiernan \& Jones, 1985; Shane \& Bashir, 1980). Rather than categorising children according to these pre-entry skills, the present study has found that children excel in modes of communication which best suit them.

Furthermore, the study has presented these modes of communication in a hierarchy of efficacy for this specific task and sample of children.

Needless to say, pre-entry skills are of some importance in the selection of augmentative systems. By considering these prerequisites, the clinician can limit a child's failures. Children with Down syndrome, for example, often encounter an unfavourable failure:success ratio in their attempts at learning (Wishart, 1993). The selection of several methods for a child to trial is likely to extend existing communication opportunities.

Providing the best possible set of strategies for communication for a child with an intellectual disability is an enormously complex task.

Augmentative procedures are not the complete solution to the communicative problems of these children. However, by a slow process of trial and assessment of these procedures, small gains can be achieved in the eventual construction of a system that allows children to communicate more effectively. The practical benefits of these procedures for children with Down syndrome (and all children with intellectual disabilities) are of great importance to the improvement of their life opportunities. By providing a mechanism, albeit a simple one, by which these children can have an impact upon their immediate environment, life opportunities for these children may be improved. The findings of this research highlight the value of a number of systems which have been shown to offer children with Down syndrome this advantage. The results offer practical directions in relation to the choice of methods of communication.

\section{Acknowledgements:}

A. Brown and M. Chiplin

\section{Correspondence:}

Associate Professor Phil Foreman

Director, Special Education Centre

University of Newcastle

Callaghan NSW

AUSTRALIA 2308 


\section{References}

Arthur, M. (1989). Augmentative communication systems for learners with severe disabilities: Towards effective assessment and placement practices. Australia and New Zealand Journal of Developmental Disabilities, 15, 119-125.

Bahrick, H. P. (1967). Relearning and the measurement of retention. Journal of Verbal Learning and Verbal Behaviour, 6, 89-94.

Benjamin, L. S. (1965). A special Latin square for the use of each subject as his own control. Psychometrika, 30, 499-513.

Berry, J. (1987). Strategies for involving parents in programs for young children using augmentative and alternative communication. Augmentative and Alternative Communication, 3, 90-94.

Bonvillian, J. D., \& Folven, R. J. (1993). Sign language acquisition. In M Marschark \& D Clark (Eds.), Psychological perspectives on deafness (pp. 229-265). Hillsdale, NJ: L. Erlbaum.

Brady, D. O., \& Smouse, A. D. (1978). A simultaneous comparison of three methods for language training with an autistic child: an experimental single case analysis. Journal of Autism and Childhood Schizophrenia, 8, 271279.

Bricker, D. D. (1972). Imitative sign training as a facilitator of word object association with low-functioning children. American Journal of Mental Deficiency, 76, 509-516.

Carr, J. (1975). Young children with Down Syndrome. London: Butterworths.

Carr, J. (1988). Six weeks to twenty-one years old: A longitudinal study of children with Down's syndrome and their families. Journal of Child Psychology and Psychiatry, 29, 407-431.

Coggins, T. E., Carpenter, R. L., \& Owings, N. O. (1983). Examining early intentional communication in Down's syndrome and nonretarded children. British Journal of Disorders of Communication, 18, 98-106.

Creedon, M. (1976). The David School: A simultaneous communication model. Paper presented at the National Society for Autistic Children Meeting, Oakbrook, Illinois.

Ferguson, G. A. (1959). Statistical analysis in psychology and education. New York: McGraw-Hill. Greenwald, C. A., \& Leonard, L. B. (1979). Communicative and sensorimotor development of Down's syndrome children. American Journal of Mental Deficiency, 84, 296-303.

Grove, N., \& Walker, M. (1990). The Makaton vocabulary: using manual signs and graphic symbols to develop interpersonal communication. Augmentative and Alternative Communication, 6, 15-28.

Hamre-Nietupski, S., Nietupski, J., \& Rathe, T. (1986). Letting the data do the talking: Selecting the appropriate nonverbal communication system for severely handicapped students. Teaching Exceptional Children, 18, 130-134.

Henderson, S. E. (1985). Motor skill development. In D. Lane \& B.Stratford (Eds.), Current approaches to Down syndrome (pp. 187-218). London: Holt, Rinehart \& Winston.

Hobson, D., \& Duncan, P. (1979). Sign learning and profoundly retarded people. Mental Retardation, 17, 3337.

Iacono, T. A., \& Duncum, J. E. (1995). Comparison of sign alone and in combination with an electronic communication device in early language intervention: Case study. Augmentative and Alternative Communication, 11, 249-259.

Iacono, T., Mirenda, P., \& Beukelman, D. (1993). Comparison of unimodal and multimodal AAC techniques for children with intellectual disabilities. Augmentative and Alternative Communication, 9, 83-94.

Iacono, T,. \& Parsons, C. (1987). Stepping beyond the teaching manuals into signing in the Real World. Australian Journal of Human Communication Disorders, 15, 101-116.

Jeanes, R. C., Reynolds, B. E., \& Coleman, B. C. (Eds.), (1993). Dictionary of Australasian signs. Victoria: Victorian School for Deaf Children.

Johnston, F., \& Stansfield, J. (1997). Expressive pragmatic skills in pre-school children with and without Down's syndrome: parental perceptions. Journal of Intellectual Disability Research, 41, 19-29. 
Kiernan, C., \& Jones, M. (1985). The heuristic programme: a combined use of sign and symbols with severely mentally retarded, autistic children. Australian Journal of Human Communication Disorders, 13, 153-168.

Konstantareas, M. (1985). Review of evidence on the relevance of sign language in the early communication training of autistic children. Australian Journal of Human Communication Disorders, 13, 77-97.

Kumin, L. (1994). Communication skills in children with Down syndrome: A guide for parents. Rockville, MD: Woodville House.

Lenneberg, E. H. (1967). Biological foundations of language. New York: John Wiley \& Sons.

Nelson, T. O. (1978). Detecting small amounts of information in memory: savings for nonrecognised items. Journal of Experimental Psychology: Learning and Memory, 4, 453-468.

Owens, R. E., \& House, L. I. (1984). Decision-making processes in augmentative communication. Journal of Speech and Hearing Disorders, 49, 18-25.

Parsons, C., \& Wills, J. (1992). Parental compliance with recommendations to utilise augmentative communication with their children with Down Syndrome. Australian Journal of Human Communication Disorders, 2, 1-19.

Prevost, P. (1983). Using the Makaton Vocabulary in early language training with a Down's baby: a single case study. Mental Handicap, 11, 28-29.

Shane, H. C., \& Bashir, A. S. (1980). Election criteria for the adoption of an augmentative communication system: Preliminary considerations. Journal of Speech and Hearing Disorders, 45, 408-414.

Sisson, L. A., \& Barrett, R. P. (1984). An alternating treatments comparison of oral and total communication training with minimally verbal retarded children. Journal of Applied Behaviour Analysis, 17, 559-566.

Sloper, P., Cunningham, C., Turner, S., \& Knussen, C. (1990). Factors related to the academic attainments of children with Down's syndrome. British Journal of Educational Psychology, 60, 284-298.

Smithers, H., \& Puffett, S. (1996). A comparison of augmentative and alternative communication systems used across regions. Proceedings of the 3rd National AGOSCI Conference. Melbourne: Australian Group on Severe Communication Impairment.

Taplin, J. E., \& Lee, M. (1982, August). The role of imitation in language acquisition. Paper presented at the Second National Child Development Conference, Melbourne.

Walker, M. (1978). The Makaton Vocabulary, in Tebbs, T., Ways and means. Basingstoke.

Walker, M. (1981). What is the Makaton Vocabulary? Special Education: Forward Trends, 3, 1-2.

Walker, M., Johnston, K., \& Cornforth, A. R. T. (1984). The revised Makaton vocabulary. In A. Cooney, J. Cowley, \& G. Knox (Eds.), Line drawings for use with the revised Makaton vocabulary (p. 4). Newcastle: Makaton Resource Centre.

Wang, P. P. \& Bellugi, U. (1994). Evidence from two genetic syndromes for a dissociation between verbal and visual-spatial short-term memory. Journal of Clinical and Experimental Neuropsychology, 16, 317-322.

Weisel, A., Dromi, E. \& Dor, S. (1990). Exploration of factors affecting attitudes towards sign language. Sign Language Studies, 68, 27-276.

Wetherby, A. M., Yonclas, D. G., \& Bryan, A. A. (1989). Communicative profiles of preschool children with handicaps: implications for early identification. Journal of Speech and Hearing Disorders, 54, 148-158.

Whitehurst, G. J., \& Vasta, R. (1975). Is language acquired through imitation? Journal of Psycholinguistic Research, 4, 37-59.

Wishart, J. G. (1993). The development of learning difficulties in children with Down's syndrome. Journal of Intellectual Disability Research, 37, 389-403. 